\title{
Network Selection in a Virtual Network Operator Environment
}

\author{
Ioannis Chamodrakas and Drakoulis Martakos \\ National and Kapodistrian University of Athens, \\ Department of Informatics and Telecommunications, Athens, Greece \\ \{ihamod, martakos\} adi.uoa.gr
}

\begin{abstract}
Service offerings of virtual network operators should focus on the needs of distinct groups of users in order to face challenges posed in the saturated telecommunications mass market. Since a virtual operator may hire capacity from a number of host networks with varied characteristics, the selection of an optimal host network according to network attributes, user preferences and QoS requirements results to the provision of services with desirable characteristics adding value for the user. Network selection in such an environment has not been studied as all previous works have focused exclusively on handover decisions in heterogeneous wireless networks. This article describes a network selection method in a virtual operator environment that uses a modified version of TOPSIS to rank alternative networks. The proposed modification enables the inclusion of QoS requirements in the ranking results. The uncertainty of user preferences is handled through the use of linguistic assessments and triangular fuzzy numbers. Simulations conducted show that the proposed approach is effective in deciding the optimum network according to all decision factors.
\end{abstract}

Keywords: network selection, virtual network operators, TOPSIS, Always Best Connected, fuzzy numbers, QoS.

\section{$1 \quad$ Introduction}

The deregulation process in the telecommunications sector has led to a vertical disintegration of the network industry [1]. Traditional network operators owned the physical network infrastructure and exercised strict control over network management and service provision. The "liberalization" of the telecommunications market led to the entry of virtual operators that carry out a subset of the functions and activities of traditional operators without possessing their own physical network and fulfill one or several roles in the value chain by hiring network capacity and basic network facilities from traditional operators under exclusive contracts [1], [2]. Virtual operators can be distinguished into three types according to their technical dependence on their "network suppliers": service providers (SPs) resell network services and products to the end-users under a different brand name, provide tariffing, billing and customer support, and undertake distribution and marketing activities; enhanced service providers (ESPs) differentiate their service offerings through the development of extra and 
innovative services; virtual network operators (VNOs) pertain to the most technically developed type of virtual operators since apart from differentiated services they also maintain their own routing infrastructure.

It has been argued that three main areas are key to VNO success: efficient and effective distribution channels to reach the target audience, retention/loyalty programs that minimize churn cost, and design of a technology road map that enables the virtual operator to quickly adopt new technologies and launch innovative products [3]. Various studies have identified as the main factor of the success of a new virtual operator entering a saturated mass market, its ability to provide differentiated service offerings in order to target specific market segments by offering value-added services that focus on the satisfaction of specific needs of distinct groups of users [2].

In this context, since a virtual operator may have established contracts with a number of traditional operators providing network capacity with different characteristics such as monetary cost, Quality of Service (QoS) attributes (bandwidth, packet loss, latency, jitter), bit/frame error rate, etc, we propose the application of the Always Best Connected concept in order to devise an intelligent network selection mechanism. The Always Best Connected concept [4], of a user being connected to a network in the best possible way based on a number of different criteria such as user preferences, size and capabilities of the service, application requirements, security, operator or corporate preferences, has been introduced in the context of heterogeneous wireless network environments, and guided the development of various vertical handover management systems [5], [6]. Vertical handover decision algorithms have been categorized into 5 groups by Kassar et al. [5], i.e. decision-function based strategies, user-centric strategies, multiple attribute decision strategies, fuzzy logic and neural network based strategies and context aware strategies, and into 4 groups by Yan et al. [6], i.e. received signal strength (RSS) based algorithms, bandwidth based algorithms, cost function based algorithms and combination based algorithms (fuzzy logic and neural network).

Inspired by the research mentioned above, this paper proposes a network selection method for the users of a virtual operator taking into account both network conditions and user preferences. This is a relatively unexplored field of research since all previous studies focused on the problem of network selection for vertical handover in the context of heterogeneous wireless environments. Apart from being an original direction for research, the proposed network selection method may be utilized by virtual operators in order to provide differentiated services according to the specific preferences and needs of the users regarding both QoS and cost of the provided services, contributing, thus, to their success in the market. The motivation of this research stems from the analysis of the requirements of an active virtual network operator in Greece and aims to be put into use after a period of simulation and real experiments.

In this article we consider QoS, monetary cost and user preferences as decision factors, Since QoS preferences are usually expressed as upper and lower limits, the structure of the selection problem fits well with the assumptions of the TOPSIS decision method. The TOPSIS method was introduced by Hwang and Yoon [7] and ranks the alternative solutions according to their distances from the zenith (the ideal alternative) and the nadir (the negative ideal alternative) points. In this case, we propose a modification of the TOPSIS method by defining the zenith point according to the QoS upper or lower limit defined for each type of service and the respective monetary costs of the network suppliers. In this way, the modified TOPSIS method produces 
results that take into account QoS requirements, enabling the treatment of different QoS profiles. The QoS profiles can be related either to specific service types (service QoS profiles) or to user-defined QoS requirements (user QoS profiles). TOPSIS has been applied with success in a number of selection/evaluation problems with a finite number of alternatives both in and out of the context of network selection [8], [9]. Moreover, the TOPSIS method is one of the best methods in addressing the problem of rank reversal [10] and its logic is consistent with the rationale of human choice. Since human judgments are uncertain with regard to the weighting of different decision criteria, many researchers have proposed fuzzy extensions of the TOPSIS method in order to grasp the vagueness of user preferences [8], [11]. For that, our method employs the use of linguistic assessments and their expression as fuzzy numbers in order to calculate the weights of different criteria. The proposed method is illustrated and validated in the article by applying it in different network selection scenarios. The simulated scenarios correspond to different service/user QoS profiles in order to observe and discuss how the proposed method would work.

\section{Technique for Order Preference by Similarity to the Ideal Solution}

The TOPSIS method ranks alternatives according to their distance from the ideal solution (zenith) and the negative ideal solution (nadir). Each alternative is represented as a point in a n-dimensional Euclidean space, and a basic assumption is that the utility of all attributes is monotonically increasing or decreasing. Thus, the zenith and nadir points are modeled as hypothetical alternatives that have respectively the best or the worst values for each attribute, from the set of attribute values of all the alternatives. The ranking principle of TOPSIS is that the best alternative is simultaneously farthest from the nadir point and closest to the zenith point in terms of Euclidean distance. In this article, we apply a modified version of TOPSIS since the zenith point is defined according to the QoS upper or lower limit of each type of service for relevant attributes according to the monotonicity of their utility.

The steps of the TOPSIS method in order to apply it to the network selection problem are the following:

1. Identify all attributes impacting the decision process as well as the alternatives under consideration. Since QoS and monetary cost preferences are taken into account as decision factors for network selection, the list of attributes may include cost per byte or cost per second of call duration (monetary cost), total bandwidth and allowed bandwidth (throughput), packet delay, packet jitter and response time (timeliness), bit error rate, packet loss, burst error and average number of retransmissions per packet (reliability), utilization, etc.

2. Construct the normalized decision matrix representing the alternatives under consideration. This step entails the collection of QoS data from the alternative networks in order to determine the respective value of each attribute under consideration.

3. Determine the weights representing the relative importance of each attribute and construct the weighted normalized decision matrix. 
4. Determine the nadir and zenith points for each type of service: the best value for each attribute is defined according to the QoS upper or lower limit and the worst value for each attribute is either the maximum or the minimum value, depending on the monotonicity of the attribute's utility. As far as the attribute of cost is concerned, the best value is the minimum cost and the worst value is the maximum cost.

5. Measure the separation $S$ of alternative networks from the nadir and zenith points using Euclidean distances. It must be noted that when an attribute value of an alternative is better than the respective value of the zenith point for a specific type of service, its superiority is ignored and the separation of the specific attribute from the zenith point is assumed to be zero.

6. Calculate the level of preference $\mathrm{C}$ of each alternative network according to the TOPSIS measure of the "relative closeness to the ideal solution"

$$
C=\frac{S_{\text {nadir }}}{S_{\text {nadir }}+S_{\text {zenith }}}
$$

7. Finally, select the network with the highest level of preference $C$.

Apart from steps 3 and 4 the procedure described above is relatively straightforward. However, step 3 entails the representation of uncertain human judgments for the determination of the attribute weights and thus crisp values are inadequate. Various researchers have proposed different fuzzy versions of TOPSIS. The fuzzification and defuzzification procedures involve the use of linguistic variables and assessments and their representation as (usually triangular) fuzzy numbers. In some cases the calculation of the distances of the fuzzified alternatives from the zenith and nadir points has been handled through the use of fuzzy distance norms, fuzzy ranking approaches or grey related analysis methods [11], [12]. In other cases, the linguistic assessments which are represented as triangular fuzzy numbers are transformed directly into crisp numbers [8], [13]. This approach is followed in order to avoid complicated fuzzy arithmetic operations and dubious fuzzy ranking approaches since it has been observed that excessive fuzzification entailing the use of complex algorithms can be considered a fallacy [13]. In this article we follow the second approach described in [8], [13].

Furthermore, step 4 entails the configuration of the QoS profiles with regard to the upper and lower limits of the QoS attribute values. QoS profiles of individual services are defined by the virtual operator, are applicable to all users and may refer to e.g. Voice service profile, Web browsing profile, Multimedia streaming profile, Messaging service profile, Dedicated data transfer profile (database backup, facsimile, etc). For example, Voice service profile upper and lower limits for packet loss are $1 \%$ to $0 \%$ and for latency are 0 to 150 milliseconds. On the other hand, Video streaming application profile upper and lower limits for packet loss and latency are $2 \%$ to $0 \%$ and 0 to 4000 milliseconds respectively. Moreover, the user may define his/her own QoS user profile applicable to all services, and thus override individual service profiles by explicitly defining upper and lower limits of the QoS attribute values. 


\section{Network Selection Scheme}

The proposed model for the network selection process in a virtual network operator environment is presented in Fig. 1. This model includes a pre-configuration stage where the virtual operator determines QoS service profiles and users identify selection attributes, provide input for the determination of their relative importance and optionally determine QoS user profiles.

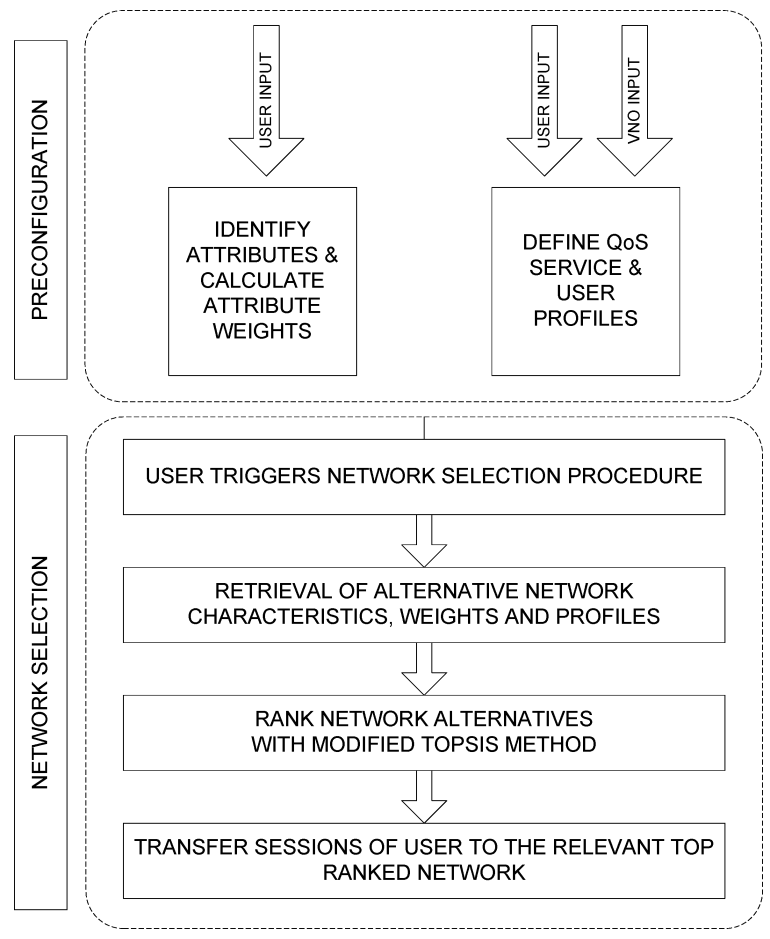

Fig. 1. Network Selection Scheme

The actual selection process is initiated by the user. Another option would be to automate the initiation step through a rule-based system that would incorporate a monitoring mechanism periodically probing the QoS network characteristics and a set of initiation rules provided by the virtual operator for each type of service. Nevertheless, we present a simplified model for the sake of the clarity of presentation since the details of such a mechanism are rather trivial.

- In the next step of the selection process, network characteristics relevant to the user-identified QoS attributes are retrieved along with attribute weights and QoS profiles.

- Then alternative networks are ranked for each type of service.

- In the last step of the selection process the sessions of the users are transferred to the top ranked network for the corresponding services. It must be noted that the 
virtual operator routing infrastructure handles the management of sessions and their transfer to the appropriate network and, therefore, it is possible to select different networks for different services without facing problems relevant to network selection in a heterogeneous wireless environment (excessive power consumption, authentication of the user terminal with multiple networks, etc.).

\section{Simulation Experiment}

In order to illustrate and validate the proposed decision method for network selection in a virtual operator environment we performed a simulation experiment. In the experiment we consider three users consuming services provided by a virtual operator that has established contracts with five host network operators supplying network capacity. Without loss of generality we have chosen five criteria to be taken into account for the network selection decision represented as attributes of the alternative networks: allowed bandwidth per user, latency, jitter, packet loss and cost per byte.

The preferences of the users for the relative importance of the criteria are given through linguistic assessments which are transformed into crisp values according to the method described in [8], [13].The linguistic assessments and the attribute weights per user are depicted in Figure. 2. The attribute values of the five alternative networks at the moment of retrieval are presented in Table 1.

Table 1. Attribute values of alternative networks

\begin{tabular}{|c|c|c|c|c|c|}
\hline Networks & $\begin{array}{c}\text { Bandwidth } \\
(\mathrm{Mbs})\end{array}$ & $\begin{array}{c}\text { Latency } \\
(\mathrm{ms})\end{array}$ & $\begin{array}{c}\text { Jitter } \\
(\mathrm{ms})\end{array}$ & $\begin{array}{c}\text { Packet } \\
\text { loss }\end{array}$ & $\begin{array}{c}\text { Cost per } \\
\text { byte }\end{array}$ \\
\hline 1 & 10 & 200 & 15 & 0,01 & 40 \\
\hline 2 & 5 & 100 & 10 & 0,005 & 50 \\
\hline 3 & 0,512 & 200 & 25 & 0,01 & 45 \\
\hline 4 & 1 & 400 & 50 & 0,02 & 30 \\
\hline 5 & 0,256 & 50 & 5 & 0,001 & 40 \\
\hline
\end{tabular}

Furthermore, we consider seven network selection scenarios corresponding to 5 service QoS profiles: voice service, video streaming, text messaging, video conferencing, web browsing and 2 user QoS profiles: Low QoS levels profile, High QoS levels profile. The modified TOPSIS method previously described is used to get the ratings and rankings of the alternative networks for each service profile. In order to compare the proposed approach with the standard TOPSIS method that does not take into account different service or user profiles we also include the ratings and rankings which standard TOPSIS produces. The upper and lower QoS limits for the chosen attributes are depicted in Fig. 3 and the results of simulations for each service profile and for the standard TOPSIS method are shown in Fig. 4.

The results depicted in Fig. 4 indicate that high discrepancies in the relative importance of the attributes influence greatly the relative rating and ranking of alternative networks, i.e. network ratings for User 2 are much different than network ratings for 
User 1 and User 3. For example, it can be seen that since User 2 weights the relative importance of the cost attribute much higher than the other attributes, it follows that Network 2 which is characterized by the highest cost per byte is rated much lower than in the case of Users 1 and 3 for all QoS profiles, whereas Network 4, which is characterized by the lowest cost, is rated much higher than in the case of Users 1 and 3 for whom it gets the lowest rating for all QoS profiles, reaching even a first ranking for User High and User Low QoS profiles. On the contrary, smaller discrepancies play a less significant role as the almost identical ratings for User 1 and User 3 show.

On the other hand, for the same relative importance of attributes, network ratings vary according to the QoS profiles due to the different service requirements pertaining to each service or user profile. For example, if User 1 is considered, it can be seen that Network 5 is optimal for Voice, Messaging and User Low QoS profiles followed by Network 2 whereas the rank order is reversed, i.e Network 2 is optimal followed by Network 5 for Video streaming, Video conferencing and User High QoS profiles.

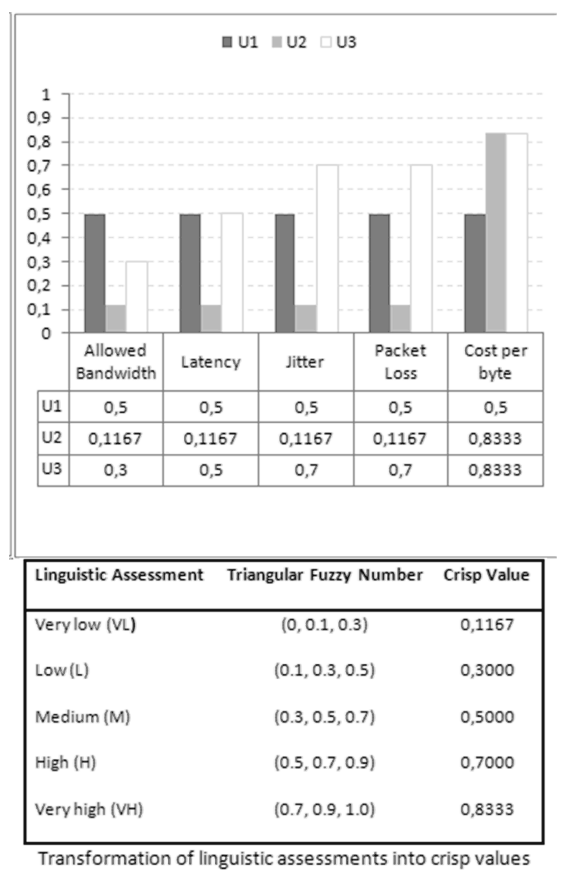

Fig. 2. Attribute weights per user / Linguistic assessment transformation 

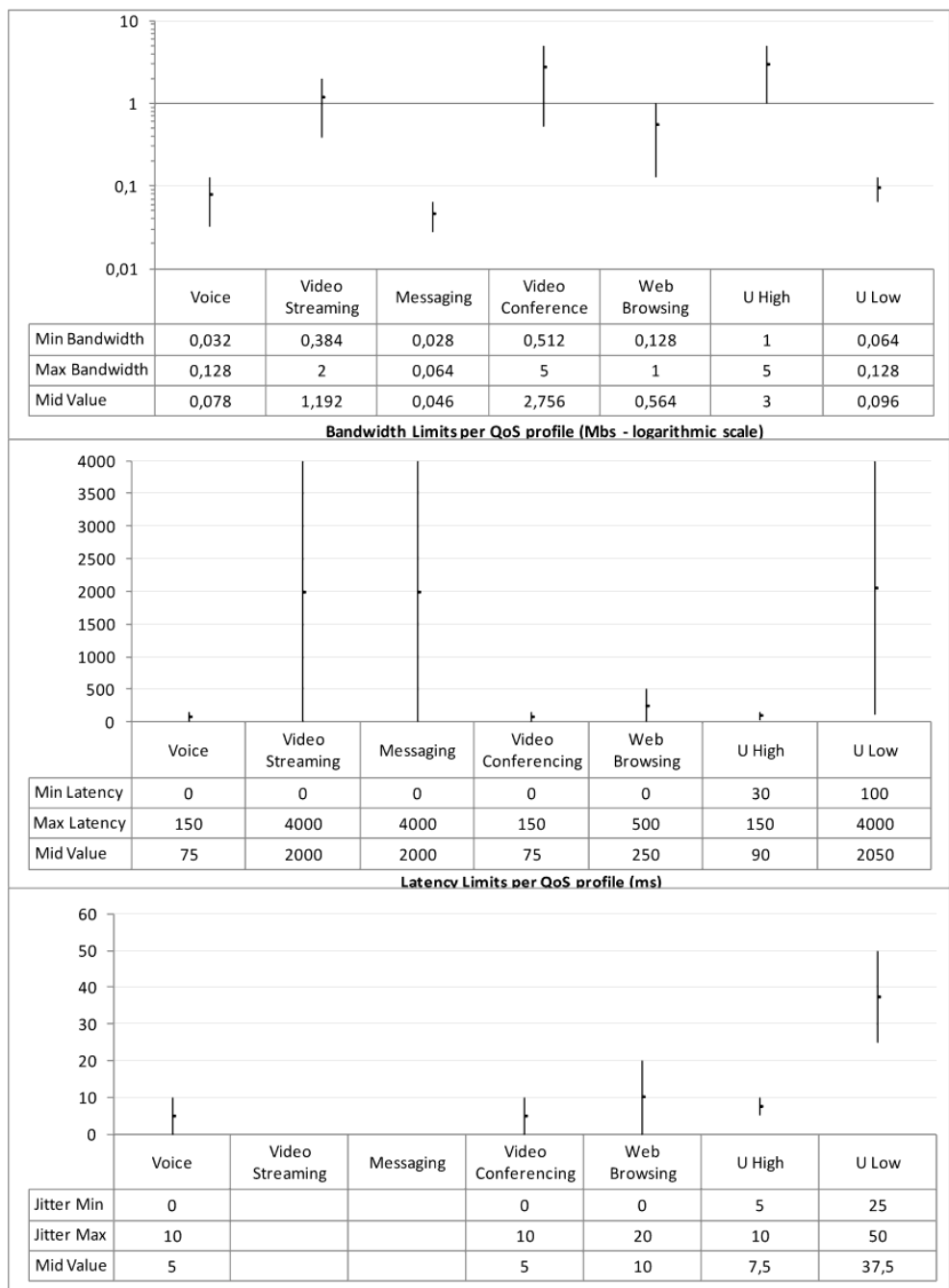

\begin{tabular}{r|c|cc} 
Mid Value & 5 & 5 & 10 \\
\hline
\end{tabular}

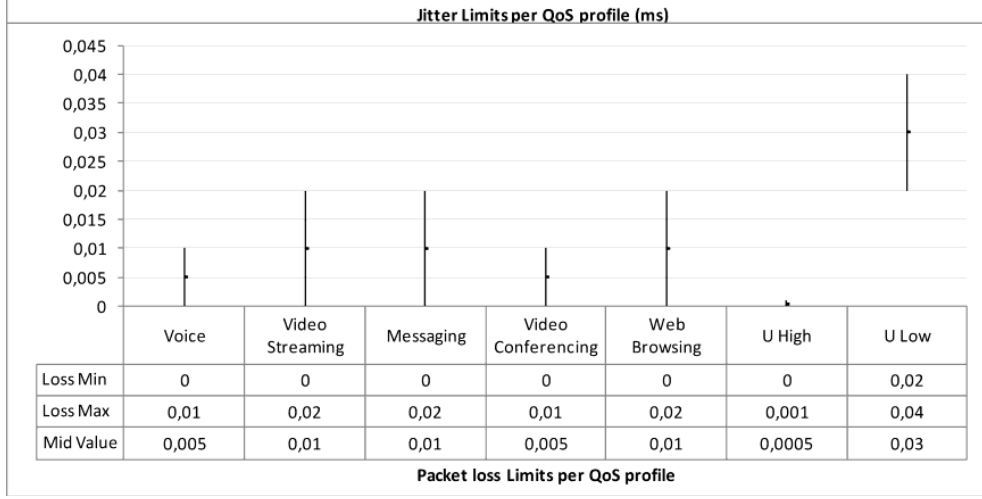

Fig. 3. QoS Profiles 

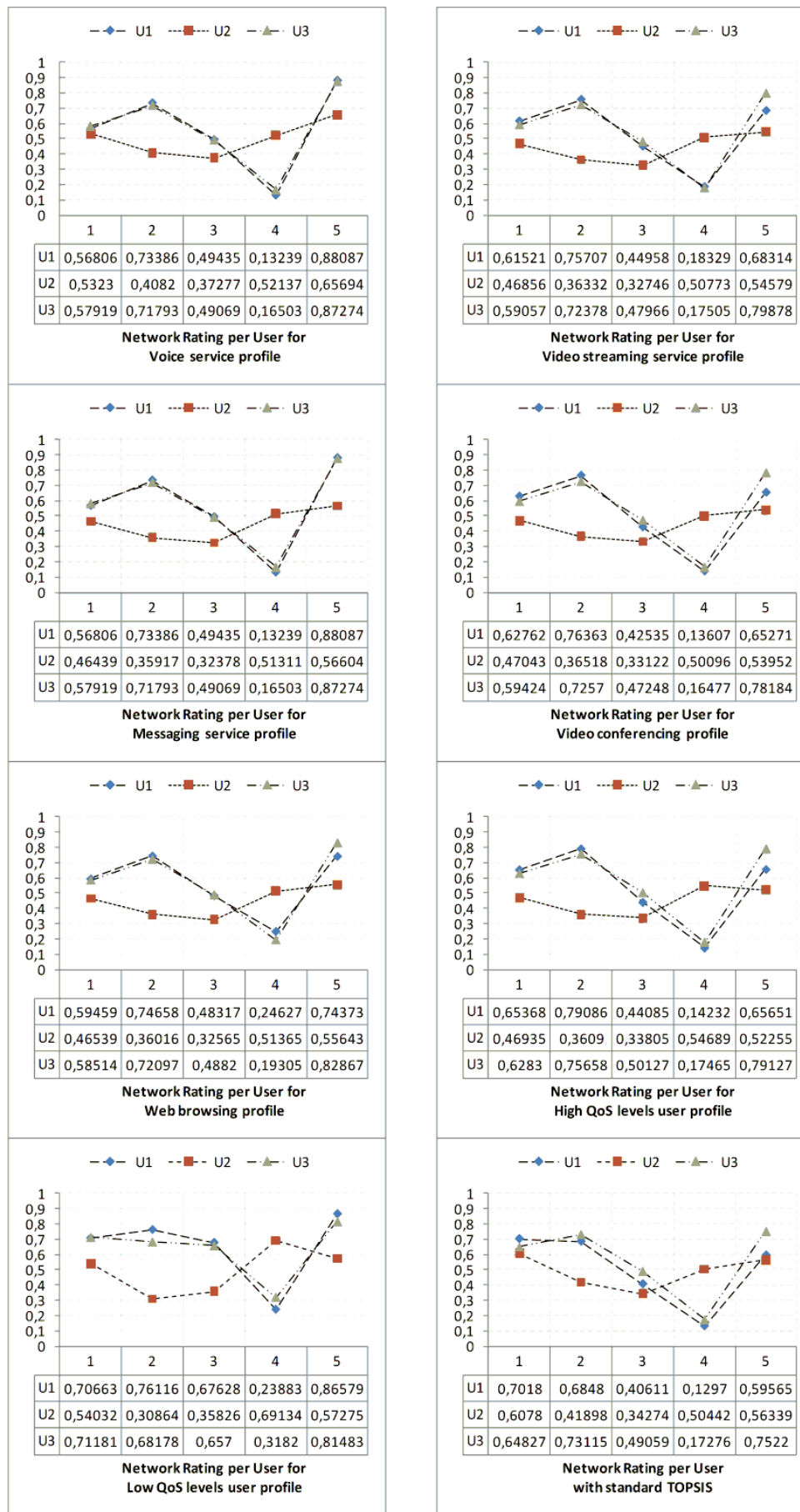

\begin{tabular}{|l|l|l|l|l|l|l|}
\hline U1 & 0,61521 & 0,75707 & 0,44958 & 0,18329 & 0,68314 \\
\hline
\end{tabular} \begin{tabular}{|l|l|l|l|l|l|} 
U2 & 0,46856 & 0,36332 & 0,32746 & 0,50773 & 0,54579 \\
\hline
\end{tabular} \begin{tabular}{l|l|l|l|l|l|} 
U3 & 0,59057 & 0,72378 & 0,47966 & 0,17505 & 0,79878 \\
\hline
\end{tabular}

Network Rating per User for Video streaming service profile

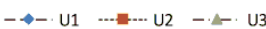

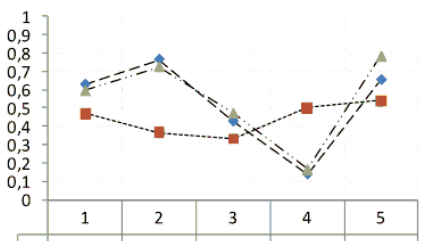

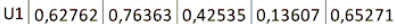
\begin{tabular}{|l|l|l|l|l|l|l|}
\hline U2 & 0,47043 & 0,36518 & 0,33122 & 0,50096 & 0,53952 \\
\hline
\end{tabular} \begin{tabular}{l|l|l|l|l|l|} 
U3 & 0,59424 & 0,7257 & 0,47248 & 0,16477 & 0,78184 \\
\hline
\end{tabular} Network Rating per User for Video conferencing profile

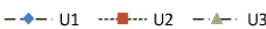

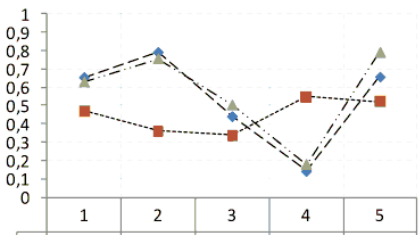

\begin{tabular}{|l|l|l|l|l|l|}
\hline U1 & 0,65368 & 0,79086 & 0,44085 & 0,14232 & 0,65651 \\
\hline
\end{tabular} \begin{tabular}{|l|l|l|l|l|l|l|}
\hline U2 & 0,46935 & 0,3609 & 0,33805 & 0,54689 & 0,52255 \\
\hline
\end{tabular} \begin{tabular}{|l|l|l|l|l|l|l|}
\hline U3 & 0,6283 & 0,75658 & 0,50127 & 0,17465 & 0,79127 \\
\hline
\end{tabular} Network Rating per User for High QoS levels user profile

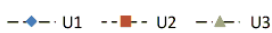

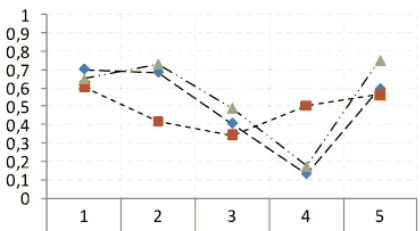

\begin{tabular}{|l|l|l|l|l|l|}
\hline U1 & 0,7018 & 0,6848 & 0,40611 & 0,1297 & 0,59565 \\
\hline
\end{tabular} \begin{tabular}{|l|l|l|l|l|l|l|}
\hline U2 & 0,6078 & 0,41898 & 0,34274 & 0,50442 & 0,56339 \\
\hline
\end{tabular} \begin{tabular}{|l|l|l|l|l|l|}
\hline U3 & 0,64827 & 0,73115 & 0,49059 & 0,17276 & 0,7522 \\
\hline
\end{tabular}

Network Rating per User with standard TOPSIS

Fig. 4. Simulation Results for seven QoS profiles 
Nevertheless, if User 3 is considered it can be seen that discrepancies in the relative importance of attributes may neutralize the effect of different service requirements, i.e. for User 3, Network 5 is optimal for all QoS profiles followed by Network 2.

As far as comparison with the standard TOPSIS method is concerned, the results of the experiments show that it is not adequate to handle selection scenarios corresponding to different QoS profiles, since it does not take into account requirements pertaining to different QoS profiles. The approach followed by Bari and Leung [9] in the context of heterogeneous wireless environments that proposes the representation of different QoS profiles through the tuning of the relative importance of attributes by the network operator is inflexible since, in the context of the same QoS profile, users cannot express their own preferences for the relative importance of attributes as they are fixed and predefined by the operator for each profile.

\section{Conclusions}

The success of a virtual network operator participating in a saturated mass market depends mainly on its ability to offer value-added services focusing on the satisfaction of specific needs of distinct groups of users. In this article we propose a novel intelligent network selection method for the users of a virtual operator that consumes network capacity with different characteristics from a number of traditional operators. Our approach constitutes an original direction for research since network selection has been studied only in the context of heterogeneous wireless networks. The proposed method takes into account both network criteria and user preferences as well as QoS profiles so that the user will consume network services in the best possible way. To this aim, the TOPSIS method is modified in order to produce results according to the QoS requirements of different types of services. Moreover, the user is situated in the center of the process not only through using linguistic assessments and triangular fuzzy numbers to capture the relative importance of network attributes according to the user preferences, but also through the ability to define user QoS profiles according to which networks are rated and ranked. This feature enhances the flexibility of the proposed mechanism in comparison with works published earlier in the context of heterogeneous wireless networks.

In order to demonstrate, validate and observe how the proposed method would work we have designed and executed a simulation experiment where three users of a virtual operator select among five host network operators in seven different scenarios corresponding to five service QoS profiles and two user QoS profiles. The simulation also facilitates a comparison of the proposed method with the standard TOPSIS method. The results of the simulation experiment show that the proposed approach is successful in producing results corresponding to different user preferences and QoS requirements.

The results of this article provide a basis for the initiation of research into the area of network selection in the environment of virtual network operators. Furthermore, the proposed method may be utilized from a virtual network operator in order to design and implement such a mechanism and provide it as an added-value service to its users. 
The main limitation of this study is that the proposed method models QoS attributes as precise, crisp data. The method should be modified in order to model these parameters as random variables in time and space which are also dependent on routes and then extract weighted averages on specific time intervals. This modification, which constitutes one of the main future directions for our research, entails the design of appropriate mechanisms for QoS measurements, which should be also implemented in user equipment. A further future direction is the evaluation of the proposed method through the consideration of other decision methods and the comparison with our approach.

\section{References}

1. Cricelli, L., Grimaldi, M., Ghiron, N.L.: The competition among mobile network operators in the telecommunication supply chain. Int. J. Prod. Econ. 131, 22-29 (2011)

2. Jaspers, F., Hulsink, W., Theeuwes, J.: Entry and Innovation in Maturing Markets: Virtual Operators in Mobile Telecommunications. Tech. Anal. Strategic Manage. 19, 205-225 (2007)

3. Bluestein, W.: Three keys to MVNO success. Telephony 247, 20 (2006)

4. Gustaffson, E., Jonsson, A.: Always Best Connected. IEEE Wirel. Commun. 10, 49-55 (2003)

5. Kassar, M., Kervella, B., Pujolle, G.: An overview of vertical handover decision strategies in heterogeneous wireless networks. Comput. Commun. 31, 2607-2620 (2008)

6. Yan, X., Sekercioglu, A., Narayanan, S.: A survey of vertical handover decision algorithms in Fourth Generation heterogeneous wireless networks. Comput. Netw. 54, 18481863 (2010)

7. Hwang, C.L., Yoon, K.: Multiple Attribute Decision Making: Methods and Applications. Springer (1981)

8. Yong, D.: Plant location selection based on fuzzy TOPSIS. Int. J. Adv. Manuf. Tech. 28, 839-844 (2006)

9. Bari, F., Leung, V.C.M.: Automated Network Selection in a Heterogeneous Wireless Network Environment. IEEE Network 21, 34-40 (2007)

10. Zanakis, S.H., Solomon, A., Wishart, N., Dublish, S.: Multi-attribute decision making: A simulation comparison of select methods. Eur. J. Oper. Res. 107, 507-529 (1998)

11. Chen, C.T.: A fuzzy approach to select location of the distribution center. Fuzzy Set. Syst. 18, 65-73 (2001)

12. Desheng Wu, J.Z., Olson, D.L.: The Method of Grey Related Analysis to Multiple Attribute Decision Making Problems with Interval Numbers. Math. Comput. Model. 42, 991-998 (2006)

13. Chamodrakas, I., Alexopoulou, N., Martakos, D.: Customer evaluation for order acceptance using a novel class of fuzzy methods based on TOPSIS. Expert Syst. Appl. 36, 7409-7415 (2009)

14. Ribeiro, R.A.: Fuzzy multiple attribute decision making: A review and new preference elicitation techniques. Fuzzy Set. Syst. 78, 155-181 (1996) 